\title{
Elemental and Phase Analysis of the Stomatopod Dactyl Club by X-Ray Mapping
}

Nicholas A. Yaraghi ${ }^{1}$, Lessa Grunenfelder ${ }^{2,3}$, Nobphadon Suksangpanya ${ }^{4}$, Nicolas Guarin ${ }^{4}$, Steven Herrera $^{1}$, Garrett Milliron ${ }^{5}$, Pablo Zavattieri ${ }^{4}$, Leigh Sheppard ${ }^{6}$, Richard Wuhrer ${ }^{6}$, David Kisailus ${ }^{1,3}$

1. Materials Science \& Engineering, University of California, Riverside, Riverside, California, USA

2. Chemical Engineering \& Materials Science, University of Southern California, Los Angeles, California, USA

3. Chemical and Environmental Engineering, University of California, Riverside, Riverside, California, USA

4. School of Civil Engineering, Purdue University, West Lafayette, Indiana, USA

5. Max Planck Institute for Colloids and Interfaces, Potsdam, Germany

6. Advanced Materials Characterization Facility, University of Western Sydney, Australia

Nature creates multifunctional composite materials by assembling organic and inorganic constituents into complex hierarchical structures [1]. The ability to precisely control the phase, morphology, and local distribution of components has resulted in a number of unique biological materials that exhibit a wide range of mechanical properties. One such structure is the raptorial appendage (dactyl club) of Odontodactylus scyllarus, a species of stomatopod (marine crustacean). The dactyl club is a multiphase bio-composite material that exhibits exceptional damage tolerance from high energy loading events. Here we examine and quantify the local distribution of elements within the dactyl club by means of XRM and quantitative EDS. We identify distinct phases, which are then correlated with ultrastructural and mechanical analyses to derive structure-function relationships as well as provide insight into the formation of an impact-resistant natural material.

Fresh dactyl clubs were obtained from both live and recently deceased specimens of $O$. scyllarus, which were maintained in an in-house sea water system. The clubs were first dissected and rinsed in DI water to remove residual salt. For XRM and EDS analyses, samples were embedded in epoxy (System 2000, Fibreglast, USA), sectioned using a low speed saw with diamond blade, and polished with progressively finer silicon carbide and diamond abrasive down to $50 \mathrm{~nm}$ grit. Polished sections were then mounted on carbon tape-coated aluminum stubs, carbon coated, and analyzed using a JEOL840 SEM operating at 20 $\mathrm{kV}$. X-ray maps were post-processed using the "Chemical Imaging” software package within the Moran Scientific Microanalysis System [2].

Figure 1 provides an overview of the dactyl club structure as well as optical and electron micrographs of a polished transverse section. Backscatter electron microscopy (BSE) in Figure 1D highlights the heavily mineralized exocuticle region, which as identified by Weaver et al., is composed of oriented hydroxyapatite [3]. Figure 1E shows the results of an $\mathrm{x}$-ray map collected at 20x magnification. The false-colored map reveals the local distribution of calcium, phosphorus, and magnesium. The map shows that the exocuticle region is highly concentrated in calcium and phosphorus, which corresponds to the crystalline calcium phosphate mineral phase present. Subsequently, the endocuticle region shows high concentrations of magnesium, which plays a role in stabilizing the amorphous calcium phosphate and calcium carbonate phases present [3]. Interestingly, there is a higher concentration of calcium located along the lateral sides of the endocuticle, indicating a more heavily mineralized sub-domain. This correlates well with the contrast observed in Figure 1D. The x-ray map also reveals a narrow phosphorus-rich region located at the interface between the endo- and exocuticle regions. This veneer 
may be important for controlling the transition from amorphous to crystalline calcium phosphate at the outer surface of the club.
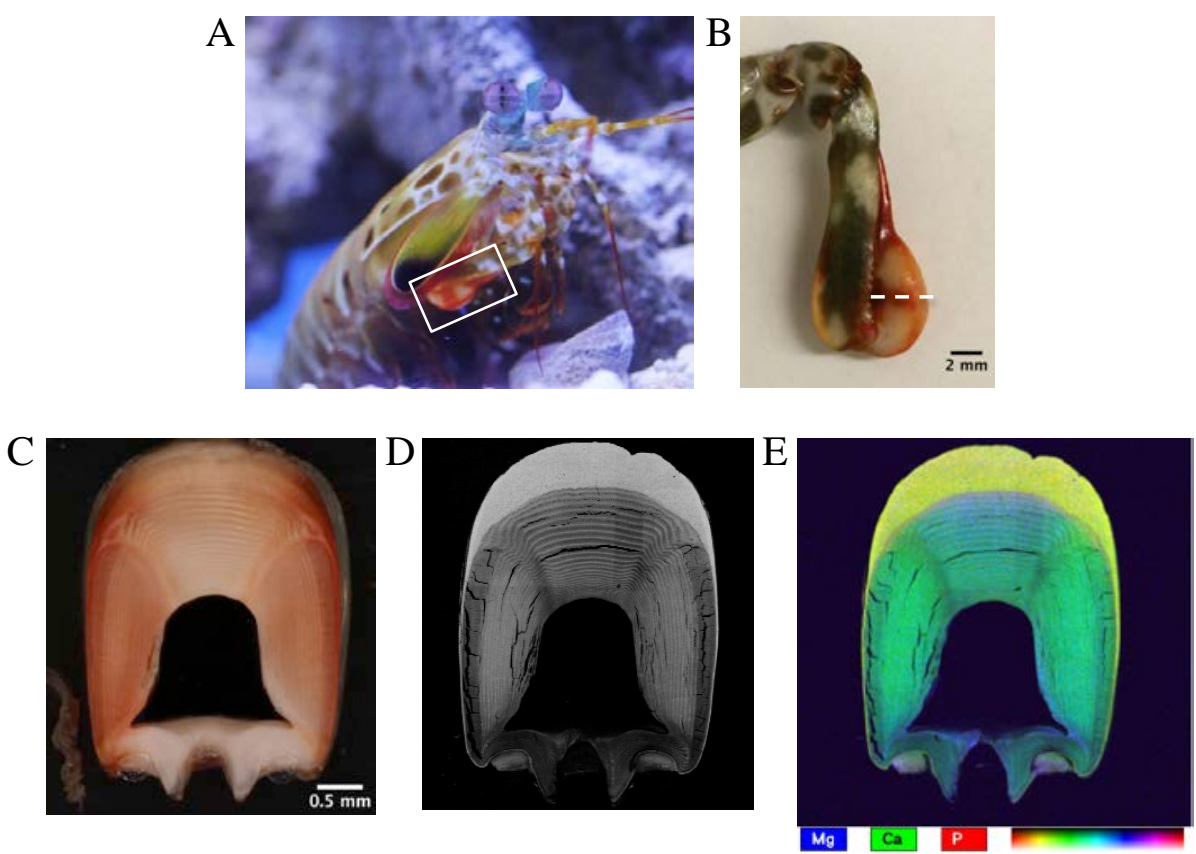

Figure 1. A) Anterior of O. scyllarus highlighting the dactyl club, which is boxed in white; B) Optical micrograph showing the raptorial appendage and dactyl segment; C) Optical micrograph of a polished transverse section of the club denoted by the dashed line in (B); D) Backscattered electron micrograph showing heavily mineralized outer surface region; E) False-colored x-ray map showing distributions of magnesium, calcium, and phosphorus throughout the club.

References:

[1] P. Fratzl, R. Weinkamer, Progress in Materials Science 52, 1263-1334 (2007)

[2] K. Moran, R. Wuhrer, Microchimica Acta 155, 209-217 (2006)

[3] Weaver et al., Science 336, 1275-1280 (2012) 